\title{
Preparation of Targeted PEG-PLGA Nanoparticles for the Treatment of Cancer Metastasis
}

\author{
Zamri Chik, Mohammed Abdullah Mahdi Alshawsh, Hasan Almoustafa
}

Department of Pharmacology, University of Malaya, Malaysia

Poly lactic-co, glycolic acid (PLGA) nanoparticles (NPs) are intensively studied as nano-carries and had been approved by US Food and drug administration for use in human. PLGA is biodegradable, and its biochemical characteristics can be controlled to suit a wild range of drug delivery and nanoparticle applications. Water soluble Polyethylene glycol (PEG) coating for nano-carriers give them a long circulation time in blood and make them invisible to the reticuloendothelial system. Doxorubicin (DOX) is a highly potent anticancer agent which is known for its highly cardiotoxic that limits its usage in chemotherapy. Hyaluronic acid is a naturally occurring polymer that binds to CD44 receptors which is overexpressed in cancer stem cells (CSC) and responsible for cancer metastasis and multi drug resistance. The objective for this study is to fabricate pegylated stealth CSC targeted PEG-PLGA-NPs using hyaluronic acid as a targeting moiety, and doxorubicin (DOX) as a drug model. Then the NPs were characterized for its chemical composition, size, zeta potential, shape, surface morphology, and encapsulation efficiency. The activity of NPs on CD44 overexpressed breast cancer cells was investigated in vitro and its efficacy on metastasis breast cancer cells will be further tested in vivo.

PEG-PLGA block copolymer was successfully fabricated using EDC-NHS chemistry, and characterised using Proton nuclear magnetic resonance (H1-NMR) and (Fourier-transform infrared spectroscopy) FT-IR. Then pegylated nanoparticles were fabricated using a nanoprecipitation method, without addition of surfactants or usage of ultrasound homogenization. The nanoparticle loaded with DOX, was coated with hyaluronic acid using EDC-NHS in water. The coating was confirmed using H1-NMR and FT-IR. The results showed that nanoparticles had a spherical shape with an average size of $100 \mathrm{~nm}$ and a zeta potential of $-15 \mathrm{mv}$ using Dynamic Light Scattering and an encapsulation efficiency of 73\% using UV-VIS spectrophotometry. Hypoxia exposed breast cancer cell line was optimized and the overexpression cells of CD44 receptors was validated using Elisa. The formulation will be tested on these cells and a validation of the targeting of hyaluronic acid coating will be achieved by comparing the IC50 of hypoxic and normoxic breast cancer cells after NPs application. 